\title{
SB-Labelings, Distributivity, and Bruhat Order on Sortable Elements
}

\author{
Henri Mühle* \\ LIAFA \\ Université Paris Diderot \\ Paris, France \\ henri.muehle@liafa.univ-paris-diderot.fr
}

Submitted: Dec 28, 2014; Accepted: May 17, 2015; Published: Jun 3, 2015

Mathematics Subject Classifications: 20F55, 06D75, 06A07

\begin{abstract}
In this article, we investigate the set of $\gamma$-sortable elements, associated with a Coxeter group $W$ and a Coxeter element $\gamma \in W$, under Bruhat order, and we denote this poset by $\mathcal{B}_{\gamma}$. We show that this poset belongs to the class of SB-lattices recently introduced by Hersh and Mészáros, by proving a more general statement, namely that all join-distributive lattices are SB-lattices. The observation that $\mathcal{B}_{\gamma}$ is join-distributive is due to Armstrong. Subsequently, we investigate for which finite Coxeter groups $W$ and which Coxeter elements $\gamma \in W$ the lattice $\mathcal{B}_{\gamma}$ is in fact distributive. It turns out that this is the case for the "coincidental" Coxeter groups, namely the groups $A_{n}, B_{n}, H_{3}$, and $I_{2}(k)$. We conclude this article with a conjectural characteriziation of the Coxeter elements $\gamma$ of said groups for which $\mathcal{B}_{\gamma}$ is distributive in terms of forbidden orientations of the Coxeter diagram.
\end{abstract}

Keywords: SB-Labeling, Möbius function, Crosscut complex, Distributive lattice, Join-distributive lattice, Antimatroids, Bruhat order, Sortable Elements, Coxeter groups

\section{Introduction}

Recently, Hersh and Mészáros introduced a new class of lattices, so-called SB-lattices [11]. They showed that these lattices admit a certain edge-labeling, which implies that the order complex of every open interval of this lattice is homotopy equivalent to a sphere or a ball

*This work was funded by the FWF Research Grant No. Z130-N13, and by a Public Grant overseen by the French National Research Agency (ANR) as part of the "Investissements d'Avenir" Program (Reference: ANR-10-LABX-0098). 
(hence the name). Equivalently, the Möbius function of such a lattice takes values only in $\{-1,0,1\}$. In the same paper they showed that every distributive lattice admits an SB-labeling, and they showed that the same is true for the weak order on a Coxeter group and for the Tamari lattice. We extend their results by showing that another class of lattices, so-called join-semidistributive lattice, belong to the class of SB-lattices as well.

Subsequently, we study a particular family of join-semidistributive lattices, namely the set of $\gamma$-sortable elements of a Coxeter groups equipped with Bruhat order. We denote this poset by $\mathcal{B}_{\gamma}$. The $\gamma$-sortable elements were defined by Reading and Speyer, see [19, 21]. To summarize, the first main result of this paper is the following.

Theorem 1. Every join-distributive lattice is an SB-lattice. In particular, the Bruhat order on $\gamma$-sortable elements is an SB-lattice for every Coxeter group $W$ and every Coxeter element $\gamma \in W$.

Join-distributivity is a generalization of distributivity, and while working with the poset $\mathcal{B}_{\gamma}$ we observed that for some Coxeter groups and for some Coxeter elements the lattice $\mathcal{B}_{\gamma}$ is in fact distributive. This led us to the question whether we can characterize the (finite) Coxeter groups $W$ and the Coxeter elements $\gamma \in W$ for which $\mathcal{B}_{\gamma}$ is distributive. We approach this problem by looking for forbidden orientations of the Coxeter diagram of $W$, and we prove the following result.

Theorem 2. Let $W$ be a finite Coxeter group. There exists a Coxeter element $\gamma \in W$ such that $\mathcal{B}_{\gamma}$ is distributive if and only if $W$ is of type $A_{n}, B_{n}, H_{3}$, or $I_{2}(k)$.

The finite Coxeter groups appearing in Theorem 2 are sometimes called the "coincidental types", since these groups enjoy a list of properties that distinguishes them from the other finite (complex) reflection groups, see [10, Theorems 8.5 and 10.2], [14, Theorem 14], [20], and [25, Remark 3.1.26]. Theorem 2 adds another property to this list. We conclude this article with a conjectural characterization of the Coxeter elements $\gamma \in W$ for which $\mathcal{B}_{\gamma}$ is distributive.

This article is organized as follows: in Section 2 we define SB-lattices, Coxeter groups and sortable elements. We subsequently prove Theorem 1 in Section 3, and define the poset $\mathcal{B}_{\gamma}$. We conclude this paper by proving Theorem 2 in Section 4 .

\section{Preliminaries}

In this section, we recall the basic concepts needed in this article. For more background on SB-labelings, we refer to [11]. For any undefined notation and additional information on Coxeter groups and sortable elements, we refer to [5] and [21], respectively.

\subsection{SB-Labelings}

Let $\mathcal{P}=(P, \leqslant)$ be a (possibly infinite) poset. An element $p \in P$ is covered by another element $q \in P$ (denoted by $p \lessdot q$ ) if $p<q$ and there exists no $z \in P$ with $p<z<q$. 
Accordingly, $q$ covers $p$ and the elements $p$ and $q$ form a cover relation or an edge in $\mathcal{P}$. The set $\mathcal{E}(\mathcal{P})=\{(p, q) \mid p \lessdot q\}$ is the Hasse diagram of $\mathcal{P}$.

For $p \leqslant q$ we call a set of the form $[p, q]=\{z \in P \mid p \leqslant z \leqslant q\}$ a (closed) interval of $\mathcal{P}$. A saturated chain in an interval $[p, q]$ is a sequence $\left(p, z_{1}, z_{2}, \ldots, z_{k-1}, q\right)$, where $z_{i} \in P$ for $i \in\{1,2, \ldots, k-1\}$ and $p \lessdot z_{1} \lessdot z_{2} \lessdot \cdots \lessdot z_{k-1} \lessdot q$.

A poset $\mathcal{P}$ is a lattice if any two elements in $\mathcal{P}$ have a least upper bound (a join) and a greatest lower bound (a meet), denoted by $\vee$ and $\wedge$, respectively. A lattice is locally finite if every interval is finite.

An edge-labeling of $\mathcal{P}$ is a map $\lambda: \mathcal{E}(\mathcal{P}) \rightarrow \Lambda$, for some set of labels $\Lambda$. An SB-labeling of a lattice $\mathcal{P}$ is an edge-labeling $\lambda$ of $\mathcal{P}$ that satisfies the following properties for every $p, p_{1}, p_{2} \in P$ with $p \lessdot p_{1}, p_{2}$ :

(i) $\lambda\left(p, p_{1}\right) \neq \lambda\left(p, p_{2}\right)$;

(ii) each saturated chain in the interval $\left[p, p_{1} \vee p_{2}\right]$ uses both labels $\lambda\left(p, p_{1}\right)$ and $\lambda\left(p, p_{2}\right)$ a positive number of times; and

(iii) none of the saturated chains in the interval $\left[p, p_{1} \vee p_{2}\right]$ uses any other label besides $\lambda\left(p, p_{1}\right)$ and $\lambda\left(p, p_{2}\right)$.

A locally finite lattice with a least element that admits an SB-labeling is called an SB-lattice.

Remark 3. In fact, the original definition of an SB-labeling given in [11, Definition 3.2] was phrased a bit differently, but it was shown in [11, Theorem 3.5] that the above definition is equivalent to the original definition.

SB-lattices enjoy the following nice property.

Theorem 4 ([11, Theorem 3.8]). The Möbius function of an SB-lattice takes values only in $\{-1,0,1\}$.

\subsection{Coxeter Groups}

A Coxeter group is a group $W$ admitting a presentation

$$
\left.W=\left\langle s_{1}, s_{2}, \ldots, s_{n}\right|\left(s_{i} s_{j}\right)^{m_{i, j}}=\varepsilon, \text { for } i, j \in\{1,2, \ldots, n\}\right\rangle,
$$

where $\varepsilon \in W$ is the identity and the numbers $m_{i, j}$ are either positive integers or the formal symbol $\infty$ for all $i, j \in\{1,2, \ldots, n\}$ such that $m_{i, j} \geqslant 2$ if $i \neq j$, and $m_{i, i}=1$. (We use the convention that $\infty$ is formally larger than every integer.) The elements in $S=\left\{s_{1}, s_{2}, \ldots, s_{n}\right\}$ are the Coxeter generators of $W$, and $n$ is the rank of $W$. A subgroup of $W$ that is generated by a subset $J \subseteq S$ is a Coxeter group in its own right and is called a standard parabolic subgroup of $W$. A Coxeter group is called irreducible if it is not isomorphic to a direct product of Coxeter groups of smaller rank. The finite irreducible Coxeter groups were completely classified by Coxeter in [6]. This classification is best visualized using so-called Coxeter diagrams. The Coxeter diagram of $W$, denoted 


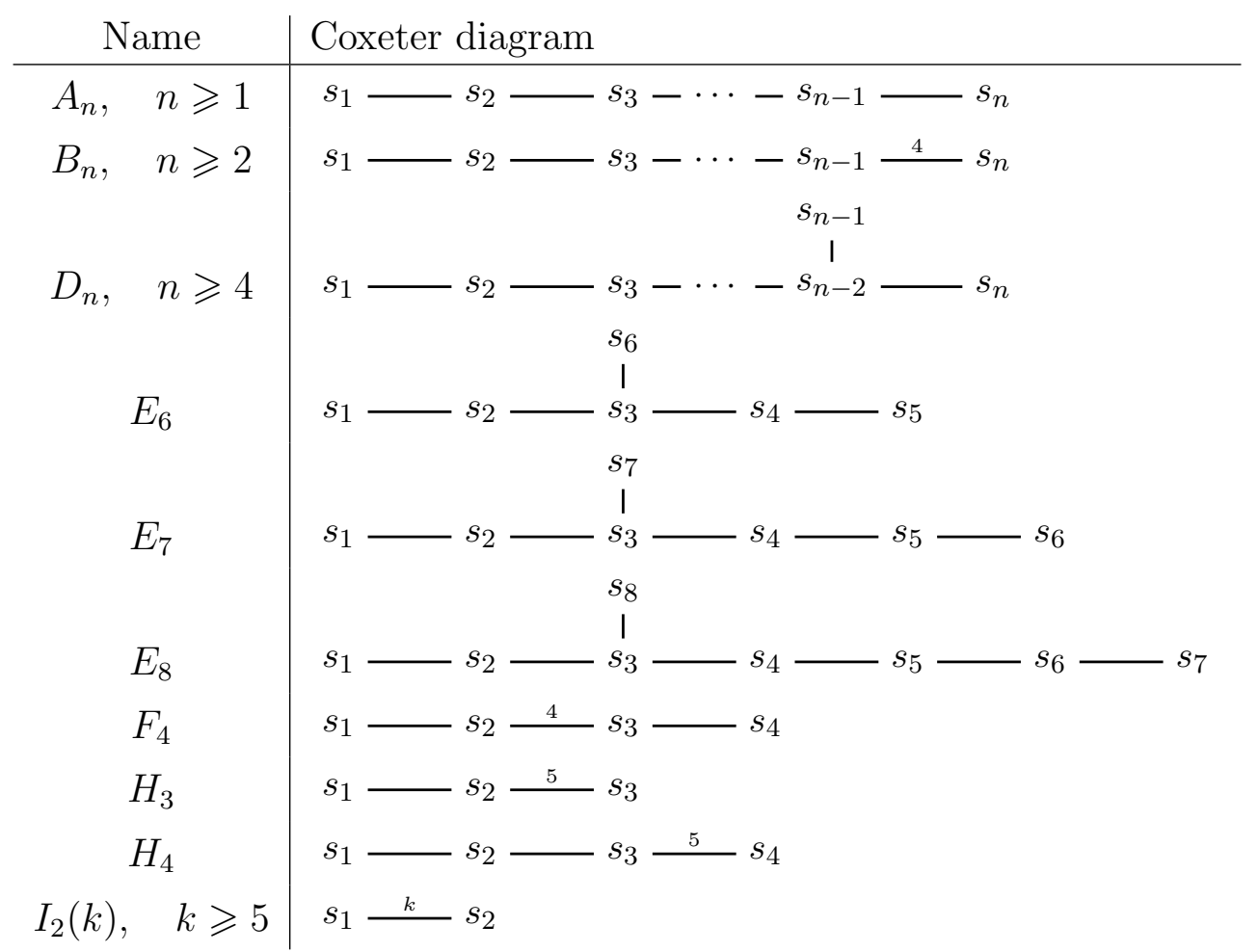

Figure 1: The Coxeter diagrams of the finite irreducible Coxeter groups.

by $\Gamma(W)$, is a labeled graph whose vertices are the Coxeter generators of $W$, and two generators $s_{i}$ and $s_{j}$ are connected by an edge if and only if $m_{i, j} \geqslant 3$. In addition, the edge between $s_{i}$ and $s_{j}$ is labeled by $m_{i, j}$ if $m_{i, j} \geqslant 4$. It is not hard to see that $W$ is irreducible if and only if its Coxeter diagram is connected. Figure 1 shows the Coxeter diagrams of the finite irreducible Coxeter groups.

Since $S$ is a generating set of $W$, we can write every $w \in W$ as a product of Coxeter generators. The least number of generators needed to form $w$ is called the Coxeter length of $w$, and will be written as $\ell_{S}(w)$. We say that a word $w=s_{i_{1}} s_{i_{2}} \cdots s_{i_{k}}$ is reduced if $\ell_{S}(w)=k$.

\section{$2.3 \quad$ Sortable Elements}

Let $W$ be a Coxeter group of rank $n$. An element $\gamma \in W$ is called a Coxeter element of $W$ if $\gamma=s_{\pi(1)} s_{\pi(2)} \cdots s_{\pi(n)}$ for some permutation $\pi$ of $\{1,2, \ldots, n\}$. Without loss of generality, we can restrict our attention to the Coxeter element $\gamma=s_{1} s_{2} \cdots s_{n}$. Consider the half-infinite word

$$
\gamma^{\infty}=s_{1} s_{2} \cdots s_{n}\left|s_{1} s_{2} \cdots s_{n}\right| s_{1} \cdots
$$

The vertical bars have no influence on the structure of the word, but shall serve for a better readability. Clearly, for every $w \in W$, every reduced word for $w$ can be written as a subword of $\gamma^{\infty}$. We call the lexicographically first subword of $\gamma^{\infty}$ that is a reduced 
word for $w$, the $\gamma$-sorting word of $w$, and we denote it by $\gamma(w)$. We can write

$$
\gamma(w)=s_{1}^{\delta_{1,1}} s_{2}^{\delta_{1,2}} \cdots s_{n}^{\delta_{1, n}}\left|s_{1}^{\delta_{2,1}} s_{2}^{\delta_{2,2}} \cdots s_{n}^{\delta_{2, n}}\right| \cdots \mid s_{1}^{\delta_{l, 1}} s_{2}^{\delta_{l, 2}} \cdots s_{n}^{\delta_{l, n}},
$$

for $l \in \mathbb{N}$ and $\delta_{i, j} \in\{0,1\}$ for $i \in\{1,2, \ldots, l\}$ and $j \in\{1,2, \ldots, n\}$. The $i$-th block of $w$ is the set $b_{i}=\left\{s_{j} \mid \delta_{i, j}=1\right\}$. We say that $w$ is $\gamma$-sortable if $b_{1} \supseteq b_{2} \supseteq \cdots \supseteq b_{l}$, and we write $C_{\gamma}$ for the set of $\gamma$-sortable elements of $W$. Further, define the set of filled positions of $w$ by

$$
\alpha_{\gamma}(w)=\left\{(i-1) n+j \mid \delta_{i, j}=1\right\} .
$$

We notice that $\alpha_{\gamma}$ depends on the choice of reduced word for $\gamma$, while $C_{\gamma}$ does not.

Remark 5. The concept of $\gamma$-sortability was introduced by Reading in [19] as a generalization of stack-sortability, and was used to define the family of Cambrian lattices associated with a Coxeter group [17, 21]. The number of $\gamma$-sortable elements of a finite Coxeter group $W$ is the $W$-Catalan number, defined in [4, Section 5.2], and Reading used the $\gamma$-sortable elements to provide a bridge between the noncrossing partitions of $W$ and the clusters of $W$, see [18].

The concept of $\gamma$-sortability has been further extended by Armstrong in [2], where he defined $\omega$-sortability for an arbitrary, not necessarily reduced word $\omega$ in the Coxeter generators of $W$, and if $\omega=\gamma^{\infty}$, then one obtains precisely the $\gamma$-sortable elements.

\section{Join-Distributive Lattices}

In this section we prove the first part of Theorem 1, namely that every join-distributive lattice admits an SB-labeling, see Theorem 7 below. Let us first recall the necessary definitions. A lattice $\mathcal{P}=(P, \leqslant)$ is meet-semidistributive if for every three elements $p, q, r \in P$ with $p \wedge q=p \wedge r$ we have $p \wedge q=p \wedge(q \vee r)$. Moreover, $\mathcal{P}$ is upper semimodular if for every two elements $p, q \in P$ with $p \wedge q \lessdot p, q$, we have $p, q \lessdot p \vee q$. Then, a lattice is join-distributive if it is both meet-semidistributive and upper semimodular. See [7] for more information on join-distributive lattices.

Recall further that an antimatroid is a pair $(M, \mathcal{F})$, where $M$ is a set and $\mathcal{F} \subseteq \wp(M)$ is a family of subsets of $M$ that satisfies the following properties:

(i) $\emptyset \in \mathcal{F} ; \quad$ and

(ii) if $X, Y \in \mathcal{F}$ with $Y \nsubseteq X$, then there exists some $x \in X \backslash Y$ such that $X \cup\{x\} \in \mathcal{F}$.

The elements of $\mathcal{F}$ are called the feasible sets of $(M, \mathcal{F})$. We have the following result.

Theorem 6 ([7, Theorem 3.3]). A lattice $\mathcal{P}$ is join-distributive if and only if there exists an antimatroid $(M, \mathcal{F})$ such that $\mathcal{P} \cong(\mathcal{F}, \subseteq)$.

In view of this correspondence we can now conclude the following result.

Theorem 7. Every join-distributive lattice admits an SB-labeling. 
Proof. Let $\mathcal{P}$ be a join-distributive lattice. In view of Theorem 6 , we can view $\mathcal{P}$ as a lattice of feasible sets of some antimatroid $(M, \mathcal{F})$, and thus every edge in $\mathcal{P}$ is determined by a pair $X, Y \in \mathcal{F}$ with $Y \backslash X=\{x\}$. This induces an edge-labeling of $\mathcal{P}$, which we will denote by $\lambda_{\mathcal{F}}$. Since $\mathcal{P}$ is upper-semimodular, it follows that for any $p, p_{1}, p_{2} \in P$ with $p \lessdot p_{1}, p_{2}$, where we write $\bar{p}=p_{1} \vee p_{2}$, we have $p_{1}, p_{2} \lessdot \bar{p}$. Since $\mathcal{P}$ is meet-semidistributive, it follows that the interval $[p, \bar{p}]$ consists only of the four elements $p, p_{1}, p_{2}, \bar{p}$. Thus we have $\lambda_{\mathcal{F}}\left(p, p_{1}\right)=\lambda_{\mathcal{F}}\left(p_{2}, \bar{p}\right)$ and $\lambda_{\mathcal{F}}\left(p, p_{2}\right)=\lambda_{\mathcal{F}}\left(p_{1}, \bar{p}\right)$, as well as $\lambda_{\mathcal{F}}\left(p, p_{1}\right) \neq \lambda_{\mathcal{F}}\left(p, p_{2}\right)$. Hence $\lambda_{\mathcal{F}}$ is an SB-labeling of $\mathcal{P}$.

Corollary 8. The Möbius function of a join-semidistributive lattice takes values only in $\{-1,0,1\}$.

Proof. This follows from Theorems 4 and 7.

Remark 9. Join-distributivity can be seen as a generalization of distributivity, see (2) and (3) below. In that sense, Theorem 7 generalizes [11, Theorem 5.1], which states that every distributive lattice is an SB-lattice.

All join-distributive lattices are by definition meet-semidistributive. Lattices that satisfy the meet-distributive law and the corresponding dual law are called semidistributive. Obviously, every distributive lattice is also semidistributive, but semidistributive lattices need no longer be graded. It is known that the Möbius function of a semidistributive lattice takes values only in $\{-1,0,1\}[8]$, and it would be interesting whether such lattices are always SB-lattices.

Another generalization of distributivity to ungraded lattices, so-called trimness, was introduced by Thomas in [24]. It is the statement of [24, Theorem 7] that the Möbius function of a trim lattice also takes values only in $\{-1,0,1\}$, and again it would be interesting to know whether trim lattices are always SB-lattices.

An important example of lattices that belong to both previously mentioned classes of lattices are Reading's Cambrian semilattices, see [21, Theorem 8.1] and [16, Theorem 1.1]. The Cambrian semilattices generalize the Tamari lattices to all Coxeter groups. Theorem 5.5 in [11] states that the Tamari lattices are SB-lattices. We could produce SBlabelings for some small Cambrian semilattices, but we could not find a uniform definition of such a labeling. We nevertheless pose the following conjecture.

Conjecture 10. Let $W$ be a Coxeter group, and let $\gamma \in W$ be a Coxeter element. The $\gamma$-Cambrian semilattice, i.e. the set $C_{\gamma}$ equipped with the weak order on $W$, is an SB-lattice.

Remark 11. Recently, McConville has investigated a slightly weaker lattice property, namely crosscut-simpliciality [13]. It follows by definition that every SB-lattice is crosscutsimplicial. He showed in particular that meet-semidistributive lattices are crosscutsimplicial, see [13, Theorem 1.2]. 


\subsection{The Bruhat Order on Sortable Elements}

In this section, we consider a special family of join-distributive lattices, namely the set of $\gamma$-sortable elements of a Coxeter group $W$ equipped with the Bruhat order, which we define next.

Definition 12. For $u, v \in W$ we write $u \leqslant_{B} v$ if and only if there exists a reduced word $v=a_{1} a_{2} \cdots a_{l}$ and indices $1 \leqslant i_{1}<i_{2}<\cdots<i_{k} \leqslant l$ such that $u=a_{i_{1}} a_{i_{2}} \cdots a_{i_{k}}$. The partial order $\leqslant_{B}$ is called the Bruhat order on $W$.

Clearly the identity $\varepsilon$ is the least element with respect to $\leqslant_{B}$. Moreover, the poset $\left(W, \leqslant_{B}\right)$ is graded by $\ell_{S}$, but it is in general not a lattice. Thus we restrict our attention to the subposet $\mathcal{B}_{\gamma}=\left(C_{\gamma}, \leqslant B\right)$, and in what follows, we index poset-theoretic notions that refer to the Bruhat order on $\gamma$-sortable elements by "B", i.e. an interval in the poset $\mathcal{B}_{\gamma}$ will be denoted by $[u, v]_{B}$, and likewise for joins and meets. Recall that a lattice is finitary if every principal order ideal is finite. In particular, finitary lattices are locally finite.

Theorem 13. The poset $\mathcal{B}_{\gamma}$ is a finitary lattice for every Coxeter group $W$ and every Coxeter element $\gamma \in W$.

Proof. First of all, let $w \in C_{\gamma}$ with $\ell_{S}(w)=k$. The interval $[\varepsilon, w]_{B}$ is certainly finite, since $w$ has finite length. Moreover, using the terminology from above, it is easy to see that $w \leqslant_{B} w^{\prime}$ if and only if $\alpha_{\gamma}(w) \subseteq \alpha_{\gamma}\left(w^{\prime}\right)$.

Let $u, u^{\prime} \in C_{\gamma}$. The word $\bar{u}$ defined by $\alpha_{\gamma}(\bar{u})=\alpha_{\gamma}(u) \cup \alpha_{\gamma}\left(u^{\prime}\right)$ is again $\gamma$-sortable. In particular, $\bar{u}$ is the least upper bound for both $u$ and $u^{\prime}$. Hence the interval $[\varepsilon, \bar{u}]_{B}$ is finite, and analogously to before we see that any two elements in this interval possess a join. Hence it is a classical lattice-theoretic result that $[\varepsilon, \bar{u}]_{B}$ is a lattice. It follows immediately that the meet of $u$ and $u^{\prime}$ exists as well, and the proof is complete.

Theorem 13 was already mentioned in [2, Section 6]. It should be remarked that in general $\mathcal{B}_{\gamma}$ is an infinite lattice without a greatest element, which implies in particular that $\mathcal{B}_{\gamma}$ is not a complete lattice. The following result is also implicit in [2].

Theorem 14 ([2]). The lattice $\mathcal{B}_{\gamma}$ is join-distributive for every Coxeter group $W$ and every Coxeter element $\gamma \in W$.

Hence the second part of Theorem 1 follows immediately from Theorem 7 .

Corollary 15. The lattice $\mathcal{B}_{\gamma}$ admits an SB-labeling for every Coxeter group $W$ and every Coxeter element $\gamma \in W$.

Proof. This follows from Theorems 7 and 14.

Proof of Theorem 1. This follows from Theorem 7 and Corollary 15.

Corollary 16. The Möbius function of $\mathcal{B}_{\gamma}$ takes values only in $\{-1,0,1\}$ for every Coxeter group $W$ and every Coxeter element $\gamma \in W$. 
The correspondence between join-distributive lattices and antimatroids allows us to associate an antimatroid with $\mathcal{B}_{\gamma}$. In general, this antimatroid is infinite, and thus the set of labels of the SB-labeling defined in the proof of Theorem 7 is potentially infinite. We notice that this labeling can be defined globally by

$$
\lambda_{\gamma}: \mathcal{E}\left(\mathcal{B}_{\gamma}\right) \rightarrow \mathbb{N}, \quad(u, v) \mapsto \alpha_{\gamma}(v) \backslash \alpha_{\gamma}(u)
$$

An analogous labeling was used in [12] to prove topological properties of the Cambrian semilattices. Now consider the map

$$
\eta: \mathbb{N} \rightarrow S, \quad i \mapsto \begin{cases}s_{n}, & \text { if } i \equiv 0 \quad(\bmod n) \\ s_{i \bmod n}, & \text { otherwise. }\end{cases}
$$

If we concatenate these two maps, then we obtain another SB-labeling of $\mathcal{B}_{\gamma}$, which in contrast to $\lambda_{\gamma}$ uses only a finite set of labels:

$$
b_{\gamma}: \mathcal{E}\left(\mathcal{B}_{\gamma}\right) \rightarrow S, \quad(u, v) \mapsto \eta\left(\alpha_{\gamma}(v) \backslash \alpha_{\gamma}(u)\right)
$$

Example 17. Figure 2 shows two Bruhat lattices labeled by the SB-labeling defined in (1). The lattice in Figure 2(a) is associated with the Coxeter group $A_{3}$ and the Coxeter element given by the oriented Coxeter diagram $s_{1} \rightarrow s_{2} \rightarrow-s_{3}$. Figure 2(b) shows the first seven ranks of the lattice associated with the affine Coxeter group $\tilde{C}_{2}$ subject to the Coxeter element given by the oriented Coxeter diagram $s_{1} \stackrel{4}{\rightarrow} s_{2} \stackrel{4}{\leftarrow} s_{3}$. (See Section 4 for an explanation of the connection between Coxeter elements and orientations of the Coxeter diagram.)

\section{Distributivity of the Bruhat Order on Sortable Elements}

Recall that a lattice $\mathcal{P}=(P, \leqslant)$ is distributive if it satisfies one of the two following, equivalent, properties for all $p, q, r \in P$ :

$$
\begin{aligned}
& p \wedge(q \vee r)=(p \wedge q) \vee(p \wedge r) \\
& p \vee(q \wedge r)=(p \vee q) \wedge(p \vee r)
\end{aligned}
$$

Armstrong remarked in [2] that for a certain Coxeter element of the Coxeter group $A_{n}$ the lattice $\mathcal{B}_{\gamma}$ coincides with the lattice of order ideals of the root poset of $A_{n}$. (For any undefined terminology, we refer once more to [5].) Hence this particular lattice is distributive. However, Armstrong remarked that this "phenomenon, unfortunately, does not persist for all types". In this section we partially answer the question for which finite Coxeter groups and which Coxeter elements the lattice $\mathcal{B}_{\gamma}$ is distributive.

Lemma 18. Let $W$ be a Coxeter group, and $\gamma \in W$ a Coxeter element. If $W^{\prime}$ is a standard parabolic subgroup of $W$ and $\gamma^{\prime} \in W^{\prime}$ denotes the restriction of $\gamma$ to $W^{\prime}$, then $\mathcal{B}_{\gamma^{\prime}}$ is an order ideal of $\mathcal{B}_{\gamma}$. 


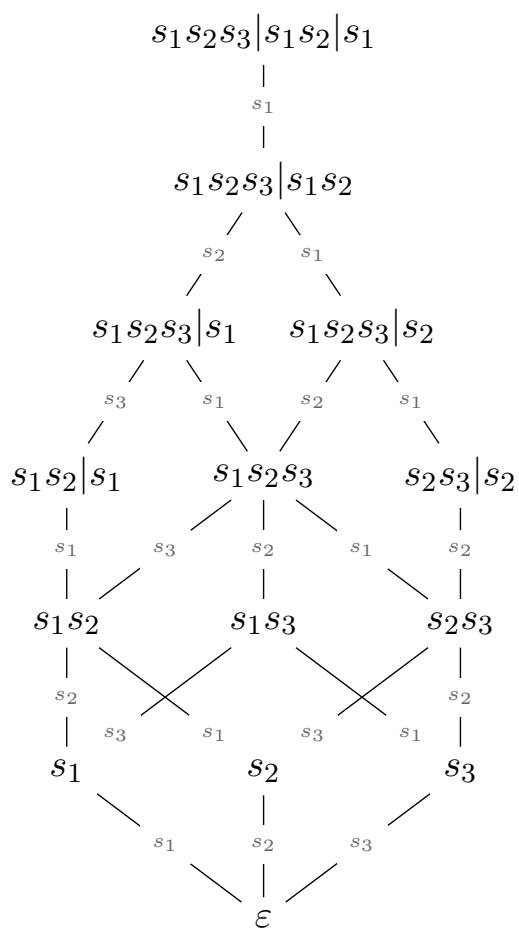

(a) $\mathcal{B}_{s_{1} s_{2} s_{3}}$ associated with $A_{3}$.

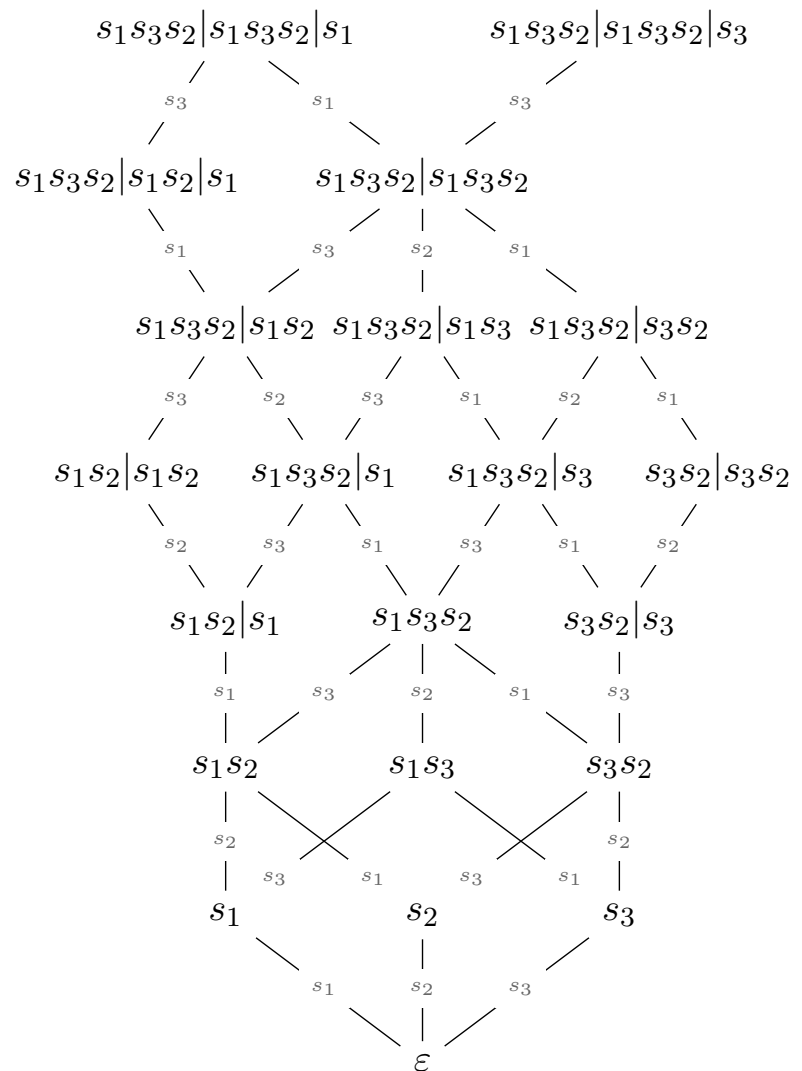

(b) The first seven ranks of $\mathcal{B}_{s_{1} s_{3} s_{2}}$ associated with $\tilde{C}_{2}$.

Figure 2: Two Bruhat lattices of sortable elements associated with the Coxeter groups $A_{3}$ and $\tilde{C}_{2}$, respectively. Their edges are labeled by the SB-labeling defined in (1).

Proof. Since $W^{\prime}$ is a subgroup of $W$, every element $w^{\prime} \in W^{\prime}$ lies in $W$ as well. Since $W^{\prime}$ is a standard parabolic subgroup of $W$, we conclude that there exists some $J \subseteq S$ such that $W^{\prime}$ is generated by $J$. Since $\gamma^{\prime}$ is the restriction of $\gamma$ to $W^{\prime}$, we conclude that $\gamma^{\prime}$ is the subword of $\gamma$ that is obtained by deleting the letters not in $J$. Hence if $w^{\prime} \in W^{\prime}$ is $\gamma^{\prime}$-sortable, then it is also $\gamma$-sortable. It follows immediately if $w \in W$ and $w^{\prime} \in W^{\prime}$ satisfy $w \leqslant_{B} w^{\prime}$, then we have $w \in W^{\prime}$. (Otherwise, the $\gamma$-sorting word of $w$ contains a letter not in $J$, which then implies $\alpha_{\gamma}(w) \nsubseteq \alpha_{\gamma}\left(w^{\prime}\right)$. This, however, contradicts $w \leqslant \leqslant_{B} w^{\prime}$.)

Remark 19. In particular, if $W$ is finite, then each standard parabolic subgroup of $W$ induces an interval of $\mathcal{B}_{\gamma}$.

Now recall that each Coxeter element $\gamma \in W$ induces an orientation $\Gamma_{\gamma}(W)$ of the Coxeter diagram of $W$ as follows: an edge between $s_{i}$ and $s_{j}$ is oriented $s_{i} \stackrel{a}{\rightarrow} s_{j}$ if and only if $s_{i}$ precedes $s_{j}$ in every reduced word for $\gamma$, see [22, Section 1.3]. The next result shows which orientations of a Coxeter diagram induce non-distributive intervals of $\mathcal{B}_{\gamma}$. 
Proposition 20. Let $W$ be a Coxeter group, let $\gamma \in W$ be a Coxeter element, and let $\Gamma_{\gamma}(W)$ be the Coxeter diagram of $W$ with the orientation induced by $\gamma$. If $\Gamma_{\gamma}(W)$ contains one of the following induced subgraphs, then $\mathcal{B}_{\gamma}$ is not distributive:

(i) $s_{i_{1}} \stackrel{a}{\leftarrow} s_{i_{2}} \stackrel{b}{\longrightarrow} s_{i_{3}}$ for $i_{1}, i_{2}, i_{3} \in\{1,2, \ldots, n\}$, and $a, b \geqslant 3$,

(ii) $s_{i_{1}} \longleftarrow s_{i_{2}} \stackrel{a}{\longleftarrow} s_{i_{3}}$ for $i_{1}, i_{2}, i_{3} \in\{1,2, \ldots, n\}$, and $a \geqslant 4$,

(iii) $s_{i_{1}} \rightarrow-s_{i_{2}} \varlimsup_{s_{i_{3}}}^{s_{i_{4}}}$ for $i_{1}, i_{2}, i_{3}, i_{4} \in\{1,2, \ldots, n\}$,

(iv) $s_{i_{1}} \rightarrow-s_{i_{2}} \underset{s_{i_{3}}}{s_{i_{4}}}$ for $i_{1}, i_{2}, i_{3}, i_{4} \in\{1,2, \ldots, n\}$,

(v) $s_{i_{1}} \rightarrow s_{i_{2}} \stackrel{a}{\rightarrow} s_{i_{3}} \leftarrow s_{i_{4}}$ for $i_{1}, i_{2}, i_{3}, i_{4} \in\{1,2, \ldots, n\}$, and $a \geqslant 4$,

(vi) $s_{i_{1}} \rightarrow s_{i_{2}} \rightarrow s_{i_{3}} \stackrel{a}{\rightarrow} s_{i_{4}}$ for $i_{1}, i_{2}, i_{3}, i_{4} \in\{1,2, \ldots, n\}$, and $a \geqslant 5$, or

(vii) $s_{i_{1}} \rightarrow s_{i_{2}} \rightarrow s_{i_{3}} \stackrel{a}{\leftarrow} s_{i_{4}}$ for $i_{1}, i_{2}, i_{3}, i_{4} \in\{1,2, \ldots, n\}$, and $a \geqslant 5$.

Proof. Suppose that $\Gamma_{\gamma}(W)$ contains an induced subgraph of form (i). Then, in particular, we have the $\gamma$-sortable elements $x=s_{i_{2}} s_{i_{1}} \mid s_{i_{2}}, y=s_{i_{2}} s_{i_{1}} s_{i_{3}}$, and $z=s_{i_{2}} s_{i_{3}} \mid s_{i_{2}}$. We have

$$
\begin{aligned}
x \wedge_{B}\left(y \vee_{B} z\right) & =x \wedge_{B} s_{i_{2}} s_{i_{1}} s_{i_{3}} \mid s_{i_{2}} \\
& =s_{i_{2}} s_{i_{1}} \mid s_{i_{2}}, \quad \text { and } \\
\left(x \wedge_{B} y\right) \vee_{B}\left(x \wedge_{B} z\right) & =s_{i_{2}} s_{i_{1}} \vee_{B} s_{i_{2}} \\
& =s_{i_{2}} s_{i_{1}},
\end{aligned}
$$

which contradicts $(2)$.

If $\Gamma_{\gamma}(W)$ contains an induced subgraph of form (ii), then consider the elements $x=$ $s_{i_{2}} s_{i_{1}} \mid s_{i_{2}}, y=s_{i_{3}} s_{i_{2}} s_{i_{1}}$, and $z=s_{i_{3}} s_{i_{2}} \mid s_{i_{3}} s_{i_{2}}$. We have

$$
\begin{aligned}
x \wedge_{B}\left(y \vee_{B} z\right) & =x \wedge_{B} s_{i_{3}} s_{i_{2}} s_{i_{1}} \mid s_{i_{3}} s_{i_{2}} \\
& =s_{i_{2}} s_{i_{1}} \mid s_{i_{2}}, \quad \text { and } \\
\left(x \wedge_{B} y\right) \vee_{B}\left(x \wedge_{B} z\right) & =s_{i_{2}} s_{i_{1}} \vee_{B} s_{i_{2}} \\
& =s_{i_{2}} s_{i_{1}}
\end{aligned}
$$

which contradicts (2).

If $\Gamma_{\gamma}(W)$ contains an induced subgraph of the form (iii), then consider the elements $x=s_{i_{4}} s_{i_{2}} s_{i_{3}} \mid s_{i_{2}}, y=s_{i_{1}} s_{i_{4}} s_{i_{2}} s_{i_{3}}$, and $z=s_{i_{1}} s_{i_{4}} s_{i_{2}} \mid s_{i_{1}} s_{i_{4}} s_{i_{2}}$. We have

$$
x \wedge_{B}\left(y \vee_{B} z\right)=x \wedge_{B} s_{i_{1}} s_{i_{4}} s_{i_{2}} s_{i_{3}} \mid s_{i_{1}} s_{i_{4}} s_{i_{2}}
$$




$$
\begin{aligned}
& =s_{i_{4}} s_{i_{2}} s_{i_{3}} \mid s_{i_{2}}, \quad \text { and } \\
\left(x \wedge_{B} y\right) \vee_{B}\left(x \wedge_{B} z\right) & =s_{i_{4}} s_{i_{2}} s_{i_{3}} \vee_{B} s_{i_{4}} s_{i_{2}} \\
& =s_{i_{4}} s_{i_{2}} s_{i_{3}},
\end{aligned}
$$

which contradicts $(2)$.

If $\Gamma_{\gamma}(W)$ contains an induced subgraph of the form (iv), then consider the elements $x=s_{i_{1}} s_{i_{3}} s_{i_{2}}\left|s_{i_{1}} s_{i_{3}} s_{i_{2}}, y=s_{i_{1}} s_{i_{4}} s_{i_{2}}\right| s_{i_{1}} s_{i_{4}} s_{i_{2}}$, and $z=s_{i_{3}} s_{i_{4}} s_{i_{2}} \mid s_{i_{3}} s_{i_{4}} s_{i_{2}}$. We have

$$
\begin{aligned}
x \wedge_{B}\left(y \vee_{B} z\right) & =x \wedge_{B} s_{i_{1}} s_{i_{3}} s_{i_{4}} s_{i_{2}} \mid s_{i_{1}} s_{i_{3}} s_{i_{4}} s_{i_{2}} \\
& =s_{i_{1}} s_{i_{3}} s_{i_{2}} \mid s_{i_{1}} s_{i_{3}} s_{i_{2}}, \quad \text { and } \\
\left(x \wedge_{B} y\right) \vee_{B}\left(x \wedge_{B} z\right) & =s_{i_{1}} s_{i_{2}}\left|s_{i_{1}} \vee_{B} s_{i_{3}} s_{i_{2}}\right| s_{i_{3}} \\
& =s_{i_{1}} s_{i_{3}} s_{i_{2}} \mid s_{i_{1}} s_{i_{3}},
\end{aligned}
$$

which contradicts $(2)$.

If $\Gamma_{\gamma}(W)$ contains an induced subgraph of the form (v), then consider the elements $x=s_{i_{2}} s_{i_{4}} s_{i_{3}}\left|s_{i_{2}} s_{i_{4}} s_{i_{3}}\right| s_{i_{2}}, y=s_{i_{4}} s_{i_{3}} \mid s_{i_{4}}$, and $z=s_{i_{1}} s_{i_{2}} s_{i_{3}}\left|s_{i_{1}} s_{i_{2}} s_{i_{3}}\right| s_{i_{1}} s_{i_{2}}$. We have

$$
\begin{aligned}
x \wedge_{B}\left(y \vee_{B} z\right) & =x \wedge_{B} s_{i_{1}} s_{i_{2}} s_{i_{4}} s_{i_{3}}\left|s_{i_{1}} s_{i_{2}} s_{i_{4}} s_{i_{3}}\right| s_{i_{1}} s_{i_{2}} \\
& =s_{i_{2}} s_{i_{4}} s_{i_{3}}\left|s_{i_{2}} s_{i_{4}} s_{i_{3}}\right| s_{i_{2}}, \quad \text { and } \\
\left(x \wedge_{B} y\right) \vee_{B}\left(x \wedge_{B} z\right) & =s_{i_{4}} s_{i_{3}}\left|s_{i_{4}} \vee_{B} s_{i_{2}} s_{i_{3}}\right| s_{i_{2}} s_{i_{3}} \\
& =s_{i_{2}} s_{i_{4}} s_{i_{3}} \mid s_{i_{2}} s_{i_{4}} s_{i_{3}},
\end{aligned}
$$

which contradicts $(2)$.

If $\Gamma_{\gamma}(W)$ contains an induced subgraph of the form (vi), then consider the elements $x=s_{i_{2}} s_{i_{3}} s_{i_{4}}\left|s_{i_{2}} s_{i_{3}} s_{i_{4}}\right| s_{i_{2}} s_{i_{3}} s_{i_{4}}\left|s_{i_{2}} s_{i_{3}} s_{i_{4}}\right| s_{i_{2}} s_{i_{3}}, y=s_{i_{2}} s_{i_{3}} s_{i_{4}}\left|s_{i_{2}} s_{i_{3}} s_{i_{4}}\right| s_{i_{2}} s_{i_{3}} s_{i_{4}}\left|s_{i_{2}} s_{i_{3}}\right| s_{i_{2}}$, and $z=s_{i_{1}} s_{i_{2}} s_{i_{3}} s_{i_{4}}\left|s_{i_{1}} s_{i_{2}} s_{i_{3}} s_{i_{4}}\right| s_{i_{1}} s_{i_{2}} s_{i_{3}} s_{i_{4}}\left|s_{i_{1}} s_{i_{2}} s_{i_{3}} s_{i_{4}}\right| s_{i_{3}}$. We have

$$
\begin{aligned}
x \wedge_{B}\left(y \vee_{B} z\right)= & x \wedge_{B} s_{i_{1}} s_{i_{2}} s_{i_{3}} s_{i_{4}}\left|s_{i_{1}} s_{i_{2}} s_{i_{3}} s_{i_{4}}\right| s_{i_{1}} s_{i_{2}} s_{i_{3}} s_{i_{4}}\left|s_{i_{1}} s_{i_{2}} s_{i_{3}} s_{i_{4}}\right| s_{i_{2}} s_{i_{3}} \\
= & s_{i_{2}} s_{i_{3}} s_{i_{4}}\left|s_{i_{2}} s_{i_{3}} s_{i_{4}}\right| s_{i_{2}} s_{i_{3}} s_{i_{4}}\left|s_{i_{2}} s_{i_{3}} s_{i_{4}}\right| s_{i_{2}} s_{i_{3}}, \quad \text { and } \quad \text { a } \\
\left(x \wedge_{B} y\right) \vee_{B}\left(x \wedge_{B} z\right)= & s_{i_{2}} s_{i_{3}} s_{i_{4}}\left|s_{i_{2}} s_{i_{3}} s_{i_{4}}\right| s_{i_{2}} s_{i_{3}} s_{i_{4}}\left|s_{i_{2}} s_{i_{3}}\right| s_{i_{2}} \\
& \vee_{B} s_{i_{2}} s_{i_{3}} s_{i_{4}}\left|s_{i_{2}} s_{i_{3}} s_{i_{4}}\right| s_{i_{2}} s_{i_{3}} s_{i_{4}} \mid s_{i_{2}} s_{i_{3}} s_{i_{4}} \\
= & s_{i_{2}} s_{i_{3}} s_{i_{4}}\left|s_{i_{2}} s_{i_{3}} s_{i_{4}}\right| s_{i_{2}} s_{i_{3}} s_{i_{4}}\left|s_{i_{2}} s_{i_{3}} s_{i_{4}}\right| s_{i_{2}},
\end{aligned}
$$

which contradicts (2).

If $\Gamma_{\gamma}(W)$ contains an induced subgraph of the form (vii), then consider the elements $x=s_{i_{2}} s_{i_{4}} s_{i_{3}}\left|s_{i_{2}} s_{i_{4}} s_{i_{3}}\right| s_{i_{2}} s_{i_{4}} s_{i_{3}}\left|s_{i_{2}} s_{i_{4}} s_{i_{3}}\right| s_{i_{2}} s_{i_{4}} s_{i_{3}}, y=s_{i_{2}} s_{i_{4}} s_{i_{3}}\left|s_{i_{2}} s_{i_{4}} s_{i_{3}}\right| s_{i_{2}} s_{i_{4}} s_{i_{3}}\left|s_{i_{2}} s_{i_{4}} s_{i_{3}}\right| s_{i_{2}}$, and $z=s_{i_{1}} s_{i_{2}} s_{i_{4}} s_{i_{3}}\left|s_{i_{1}} s_{i_{2}} s_{i_{4}} s_{i_{3}}\right| s_{i_{1}} s_{i_{2}} s_{i_{4}} s_{i_{3}}\left|s_{i_{1}} s_{i_{2}} s_{i_{4}} s_{i_{3}}\right| s_{i_{4}} s_{i_{3}}$. We have

$$
\begin{aligned}
& x \wedge_{B}\left(y \vee_{B} z\right)=x \wedge_{B} s_{i_{1}} s_{i_{2}} s_{i_{4}} s_{i_{3}}\left|s_{i_{1}} s_{i_{2}} s_{i_{4}} s_{i_{3}}\right| s_{i_{1}} s_{i_{2}} s_{i_{4}} s_{i_{3}}\left|s_{i_{1}} s_{i_{2}} s_{i_{4}} s_{i_{3}}\right| s_{i_{2}} s_{i_{4}} s_{i_{3}} \\
& =s_{i_{2}} s_{i_{4}} s_{i_{3}}\left|s_{i_{2}} s_{i_{4}} s_{i_{3}}\right| s_{i_{2}} s_{i_{4}} s_{i_{3}}\left|s_{i_{2}} s_{i_{4}} s_{i_{3}}\right| s_{i_{2}} s_{i_{4}} s_{i_{3}} \text {, and } \\
& \left(x \wedge_{B} y\right) \vee_{B}\left(x \wedge_{B} z\right)=s_{i_{2}} s_{i_{4}} s_{i_{3}}\left|s_{i_{2}} s_{i_{4}} s_{i_{3}}\right| s_{i_{2}} s_{i_{4}} s_{i_{3}}\left|s_{i_{2}} s_{i_{4}} s_{i_{3}}\right| s_{i_{2}} \\
& \vee_{B} s_{i_{2}} s_{i_{4}} s_{i_{3}}\left|s_{i_{2}} s_{i_{4}} s_{i_{3}}\right| s_{i_{2}} s_{i_{4}} s_{i_{3}}\left|s_{i_{2}} s_{i_{4}} s_{i_{3}}\right| s_{i_{4}} \\
& =s_{i_{2}} s_{i_{4}} s_{i_{3}}\left|s_{i_{2}} s_{i_{4}} s_{i_{3}}\right| s_{i_{2}} s_{i_{4}} s_{i_{3}}\left|s_{i_{2}} s_{i_{4}} s_{i_{3}}\right| s_{i_{2}} s_{i_{4}} \text {, }
\end{aligned}
$$

which contradicts (2). 
We obtain the following corollary immediately.

Corollary 21. If $W=D_{n}$, for $n \geqslant 4, W=E_{n}$, for $n \in\{6,7,8\}, W=F_{4}$, or $W=H_{4}$, and $\gamma \in W$ is a Coxeter element, then $\mathcal{B}_{\gamma}$ is not distributive.

Proof. First consider $W=D_{4}$. The eight orientations of $\Gamma\left(D_{4}\right)$ are shown below.

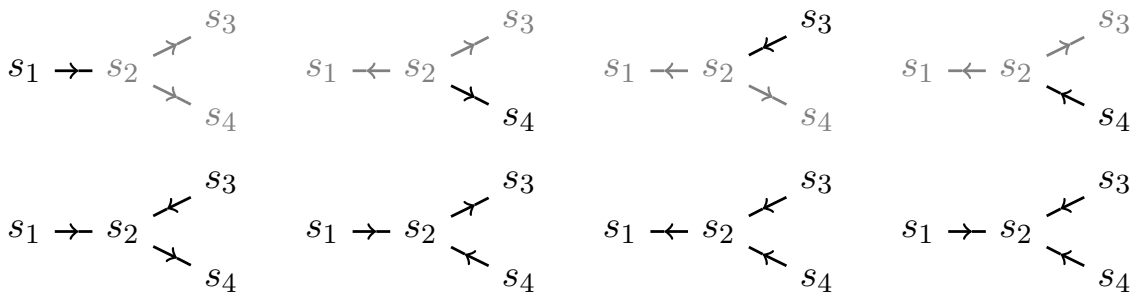

The first four orientations in the first row correspond to case (i) in Proposition 20, the first three orientations in the second row correspond to case (iii) in Proposition 20, and the fourth orientation in the second row corresponds to case (iv) in Proposition 20. Hence $\mathcal{B}_{\gamma}$ cannot be distributive for Coxeter elements inducing these orientations.

If $W=D_{n}$, for $n>4$, or $W=E_{n}$, for $n \in\{6,7,8\}$, then we conclude from Figure 1 that $W$ has a standard parabolic subgroup isomorphic to $D_{4}$. In view of Lemma 18 and Remark 19, we conclude that $\mathcal{B}_{\gamma}$ contains a non-distributive interval, and hence cannot be distributive itself.

Now let $W=F_{4}$. The eight orientations of $\Gamma\left(F_{4}\right)$ are shown below.

$$
\begin{array}{lll}
s_{1} \rightarrow s_{2} \stackrel{4}{\leftarrow} s_{3} \rightarrow-s_{4} & s_{1} \leftarrow s_{2} \stackrel{4}{\rightarrow} s_{3} \rightarrow s_{4} & s_{1} \leftarrow s_{2} \stackrel{4}{\rightarrow-} s_{3} \leftarrow s_{4} \\
s_{1} \leftarrow s_{2} \stackrel{4}{\leftarrow} s_{3} \rightarrow-s_{4} & s_{1} \leftarrow s_{2} \stackrel{4}{\leftarrow} s_{3} \leftarrow s_{4} & s_{1} \rightarrow s_{2} \stackrel{4}{\rightarrow} s_{3} \rightarrow s_{4} \\
s_{1} \rightarrow-s_{2} \stackrel{4}{\rightarrow} s_{3} \leftarrow s_{4} & s_{1} \rightarrow s_{2} \stackrel{4}{\leftarrow} s_{3} \leftarrow s_{4} &
\end{array}
$$

The first four orientations correspond to case (i) in Proposition 20, the last two orientations in the second row correspond to case (ii) in Proposition 20, and the two orientations in the third row correspond to case (v) in Proposition 20. Hence $\mathcal{B}_{\gamma}$ cannot be distributive for Coxeter elements inducing these orientations.

Now let $W=H_{4}$. The eight orientations of $\Gamma\left(H_{4}\right)$ are shown below.

$$
\begin{array}{lll}
s_{1} \rightarrow s_{2} \leftarrow s_{3} \stackrel{5}{\rightarrow} s_{4} & s_{1} \leftarrow s_{2} \rightarrow s_{3} \stackrel{5}{\rightarrow} s_{4} & s_{1} \leftarrow s_{2} \rightarrow s_{3} \stackrel{5}{\leftarrow} s_{4} \\
s_{1} \leftarrow s_{2} \leftarrow s_{3} \stackrel{5}{\rightarrow} s_{4} & s_{1} \leftarrow s_{2} \leftarrow s_{3} \stackrel{5}{\leftarrow} s_{4} & s_{1} \rightarrow s_{2} \leftarrow s_{3} \stackrel{5}{\leftarrow} s_{4} \\
s_{1} \rightarrow s_{2} \rightarrow s_{3} \stackrel{5}{\rightarrow} s_{4} & s_{1} \rightarrow s_{2} \rightarrow s_{3} \stackrel{5}{\leftarrow} s_{4} &
\end{array}
$$

The first four orientations correspond to case (i) in Proposition 20, and the last two orientations in the second row correspond to case (ii) in Proposition 20. The first orientation in the third row corresponds to case (vi) in Proposition 20, and the second orientation in the third row corresponds to case (vii) in Proposition 20. 
Proposition 22. If $W=A_{n}$ for $n \geqslant 1, W=B_{n}$ for $n \geqslant 2, W=H_{3}$ or $W=I_{2}(k)$ for $k \geqslant 5$, then there exists a Coxeter element $\gamma \in W$ such that $\mathcal{B}_{\gamma}$ is distributive.

Proof. Let $W=A_{n}$ and let $\gamma$ be the Coxeter element that induces the linear orientation $s_{1} \rightarrow s_{2} \rightarrow \cdots \rightarrow s_{n}$. It follows from the bijection in [3] that $\mathcal{B}_{\gamma}$ is isomorphic to the lattice of classical Dyck paths under dominance order. This lattice is known to be distributive, see for instance [9, Corollary 2.2]. The same bijection implies also that $\mathcal{B}_{\gamma}$ is isomorphic to the lattice of order ideals of the root poset of $A_{n}$.

Let $W=B_{n}$ and let $\gamma$ be the Coxeter element that induces the linear orientation $s_{1} \rightarrow-s_{2} \rightarrow \cdots \rightarrow-s_{n-1} \stackrel{4}{\rightarrow} s_{n}$. It follows from the bijection in [23, Section 3] that $\mathcal{B}_{\gamma}$ is isomorphic to the lattice of type- $B$ Dyck paths under dominance order. This lattice is known to be distributive, see [15, Theorem 2.9]. The same bijection implies also that $\mathcal{B}_{\gamma}$ is isomorphic to the lattice of order ideals of the root poset of $B_{n}$.

Let $W=I_{2}(k)$ for $k \geqslant 5$, and denote by $s_{1}$ and $s_{2}$ the Coxeter generators of $W$. We have $\mathcal{B}_{s_{1} s_{2}} \cong \mathcal{B}_{s_{2} s_{1}}$, and this lattice is trivially distributive. It is also isomorphic to the lattice of order ideals of the "root poset" of $I_{2}(k)$ defined by Armstrong in [1, Figure 5.15].

Let $W=H_{3}$ and let $\gamma$ be the Coxeter element that induces the linear orientation $s_{1} \rightarrow-s_{2} \stackrel{5}{\rightarrow} s_{3}$. We can easily check by computer that the resulting lattice $\mathcal{B}_{\gamma}$ is distributive. However, in this case, $\mathcal{B}_{\gamma}$ is not isomorphic to the lattice of order ideals of the "root poset" of $\mathrm{H}_{3}$ defined by Armstrong in [1, Figure 5.15].

Proof of Theorem 2. This follows immediately from Proposition 22.

We conclude this section with the following conjecture.

Conjecture 23. For finite Coxeter groups, the list in Proposition 20 is exhaustive, i.e. if $W$ is a finite Coxeter group, $\gamma \in W$ is a Coxeter element and the orientation $\Gamma_{\gamma}(W)$ of the Coxeter diagram of $W$ induced by $\gamma$ does not contain one of the induced subgraphs listed in Proposition 20, then $\mathcal{B}_{\gamma}$ is distributive.

Remark 24. The claim of Conjecture 23 for $W=H_{3}$ can be verified by computer. For $W=B_{3}$, the only orientation other than the one in Proposition 22 that is conjectured to yield a distributive lattice $\mathcal{B}_{\gamma}$ is $s_{1} \rightarrow-s_{2} \rightarrow-\cdots \rightarrow s_{n-1} \stackrel{4}{\leftarrow} s_{n}$. For $W=A_{n}$ there are several more options.

\section{References}

[1] Drew Armstrong. Generalized Noncrossing Partitions and Combinatorics of Coxeter Groups. Memoirs of the American Mathematical Society, 202, 2009.

[2] Drew Armstrong. The Sorting Order on a Coxeter Group. Journal of Combinatorial Theory (Series A), 116:1285-1305, 2009.

[3] Jason Bandlow and Kendra Killpatrick. An Area-to-Inv Bijection Between Dyck Paths and 312-avoiding Permutations. The Electronic Journal of Combinatorics, 8:\#R40, 2001. 
[4] David Bessis. The Dual Braid Monoid. Annales Scientifiques de l'École Normale Supérieure, 36:647-683, 2003.

[5] Anders Björner and Francesco Brenti. Combinatorics of Coxeter Groups. Springer, New York, 2005.

[6] Harold S. M. Coxeter. The Complete Enumeration of Finite Groups of the Form $R_{i}^{2}=\left(R_{i} R_{j}\right)^{k_{i j}}=1$. Journal of the London Mathematical Society, 10:21-25, 1935.

[7] Paul H. Edelman. Meet-Distributive Lattices and the Anti-Exchange Closure. Algebra Universalis, 10:290-299, 1980.

[8] Jonathan D. Farley, 2013. Personal Communication.

[9] Luca Ferrari and Renzo Pinzani. Lattices of Lattice Paths. Journal of Statistical Planning and Inference, 135:77-92, 2005.

[10] Sergey Fomin and Nathan Reading. Generalized Cluster Complexes and Coxeter Combinatorics. International Mathematics Research Notices, 44:2709-2757, 2005.

[11] Patricia Hersh and Karola Mészáros. SB-Labelings and Posets with each Interval Homotopy Equivalent to a Sphere or a Ball. Preprint, 2014. arXiv:1407.5311.

[12] Myrto Kallipoliti and Henri Mühle. On the Topology of the Cambrian Semilattices. The Electronic Journal of Combinatorics, 20:\#P48, 2013.

[13] Thomas McConville. Crosscut-Simplicial Lattices. Preprint, 2014. arXiv:1409.6269

[14] Alex R. Miller. Foulkes Characters for Complex Reflection Groups. to appear in Proceedings of the American Mathematical Society, 2015.

[15] Henri Mühle. A Heyting Algebra on Dyck Paths of Type A and B. Preprint, 2013. arXiv: 1312.0551

[16] Henri Mühle. Trimness of Closed Intervals in Cambrian Semilattices. Preprint, 2015. arXiv: 1501.02619

[17] Nathan Reading. Cambrian Lattices. Advances in Mathematics, 205:313-353, 2006.

[18] Nathan Reading. Clusters, Coxeter-Sortable Elements and Noncrossing Partitions. Transactions of the American Mathematical Society, 359:5931-5958, 2007.

[19] Nathan Reading. Sortable Elements and Cambrian Lattices. Algebra Universalis, 56:411-437, 2007.

[20] Nathan Reading. Chains in the Noncrossing Partition Lattice. SIAM Journal on Discrete Mathematics, 22:875-886, 2008.

[21] Nathan Reading and David E. Speyer. Sortable Elements in Infinite Coxeter Groups. Transactions of the American Mathematical Society, 363:699-761, 2011.

[22] Jian-yi Shi. The Enumeration of Coxeter Elements. Journal of Algebraic Combinatorics, 6:161-171, 1997.

[23] Christian Stump. More Bijective Catalan Combinatorics on Permutations and Signed Permutations. Journal of Combinatorics, 4:419-447, 2013.

[24] Hugh Thomas. An Analogue of Distributivity for Ungraded Lattices. Order, 23:249269, 2006.

[25] Nathan Williams. Cataland. Dissertation, 2013. 